\title{
Dynamic Nanoscale Imaging of Beam Sensitive Materials with In Situ Fluid Stage
}

\author{
K.L. Jungjohann,* J.E. Evans, ${ }^{* *}$ T.B. LaGrange, ${ }^{* * *}$ M. Wall,*** M.K. Santala, ${ }^{* * *}$ B.W. Reed,*** \\ G.H. Campbell, *** and N.D. Browning****** \\ * Department of Chemical Engineering and Materials Science, University of California, One Shields \\ Avenue, Davis, California 95616 \\ ** Department of Molecular and Cellular Biology, University of California, One Shields Avenue, \\ Davis, California 95616 \\ *** Lawrence Livermore National Laboratory, 7000 East Avenue, Livermore, California 94550
}

In effort to solve biological processes at the molecular level, the dynamic transmission electron microscope (DTEM) has been used in combination with an in situ fluid stage holder. The DTEM has proven its utility by imaging material processes such as phase transformations with spatial resolutions of about $10 \mathrm{~nm}$ and time resolutions of 15ns.[1] In this experiment the dynamic transmission electron microscopy techniques are applied to solution systems using a fluid stage holder. The holder consists of $50 \mathrm{~nm}$ thick electron transparent $\mathrm{Si}_{3} \mathrm{~N}_{4}$ windows separated by $0.5 \mu \mathrm{m}$ $\mathrm{Au}$ platforms, $4 \mathrm{~nm}$ spatial resolution has been demonstrated with a fluid stage in scanning transmission electron microscopy (STEM).[2] The primary advantage of the DTEM over STEM imaging is the capability of acquiring signals on ultrafast time scales. Under this condition the effects of Brownian motion are reduced, and do not contribute to image blur. This is especially useful for easily damaged biological specimens, as image is captured by the nanosecond pulse before damage occurs and the fluid flow inside the cell extracts the damaged structures created from preceding electron beam interactions. In particular, membrane protein imaging could benefit from this technique.

Gold nanoparticles were used for comparison of the dynamic imaging to conventional cryoelectron microscopy and negative stain techniques that image static biomaterials. Scattering of the electron beam through the fluid cell in combination with space charge effects from the high current electron beam of the DTEM have degraded the signal to noise ratio. General biological processes require higher spatial resolution in comparison to temporal resolution, providing a tradeoff to increase the resolvable characteristics within the sample. The signal to noise ratios achieved with DTEM are comparable to those in standard cryo-EM imaging, but with the added benefit of very high time resolution and the capability to image structures before damage can occur.

\section{References}

[1] T.B. LaGrange et al., Ultramicroscopy. 108 (2008) 1441.

[2] N. de Jonge et al., PNAS. 106, 7 (2009) 2159.

[3] M.R. Armstrong, Ultramicroscopy. 107 (2007) 356.

[4] Research performed under the auspices of the U.S. Department of Energy, Office of Basic Energy Sciences, Divisions of Materials Sciences and Engineering and Chemical Sciences by Lawrence Livermore National Laboratory under contract DE-AC52-07NA27344 and National Institutes of Health by University California Davis grant number R01GM091755-01. 


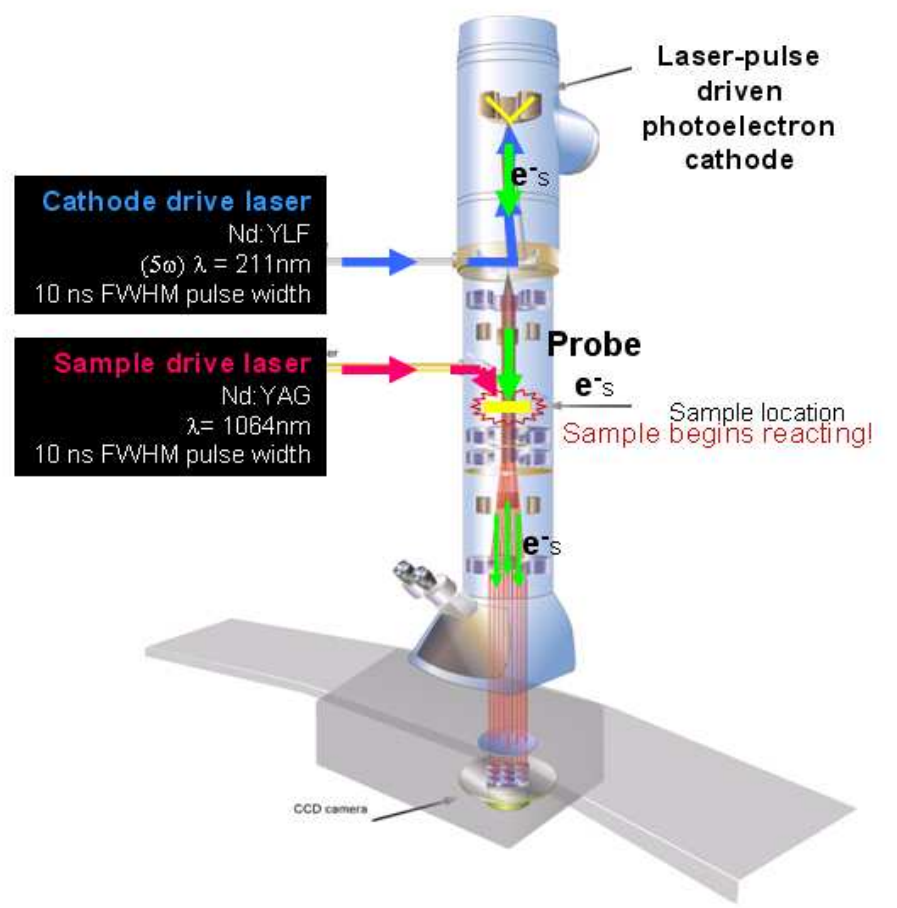

FIG. 1. Dynamic Transmission Electron Microscope: JEOL 2000FX column with two laser ports into the column.[1,3] The cathode drive laser stimulates a photoelectron cathode for emission pulse of $10^{9}$ electrons; sample drive laser interacts directly with the sample for reaction initiation.

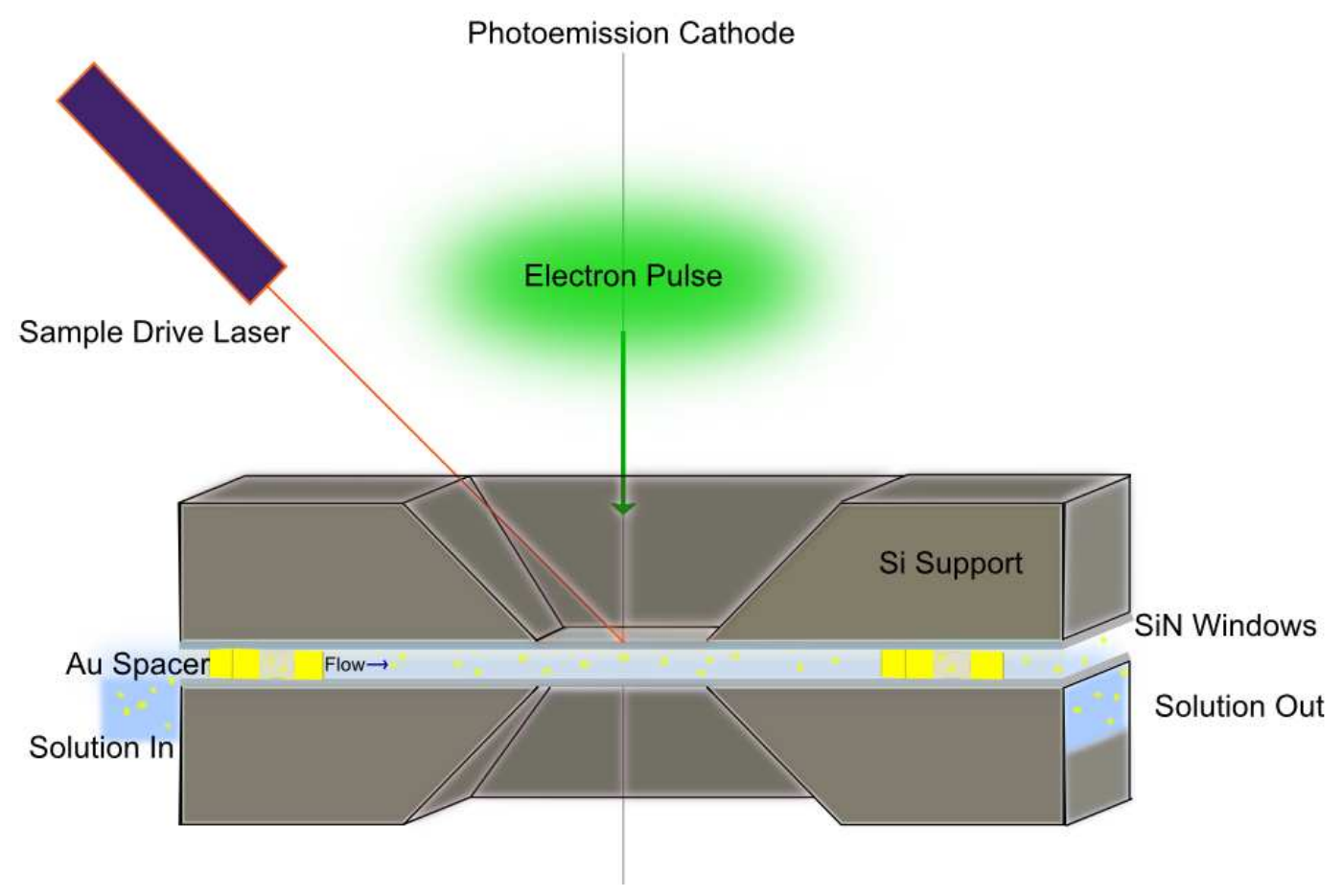

FIG. 2. Dynamic transmission electron microscope imaging, used in combination with the fluid stage holder. A cross-section view of a fluid cell flowing Au nanoparticles between two $50 \mathrm{~nm}$ thick $\mathrm{SiN}$ membrane windows spaced with $500 \mathrm{~nm}$ Au platforms. 